\section{Tender nodules on the palms and soles after chemotherapy}

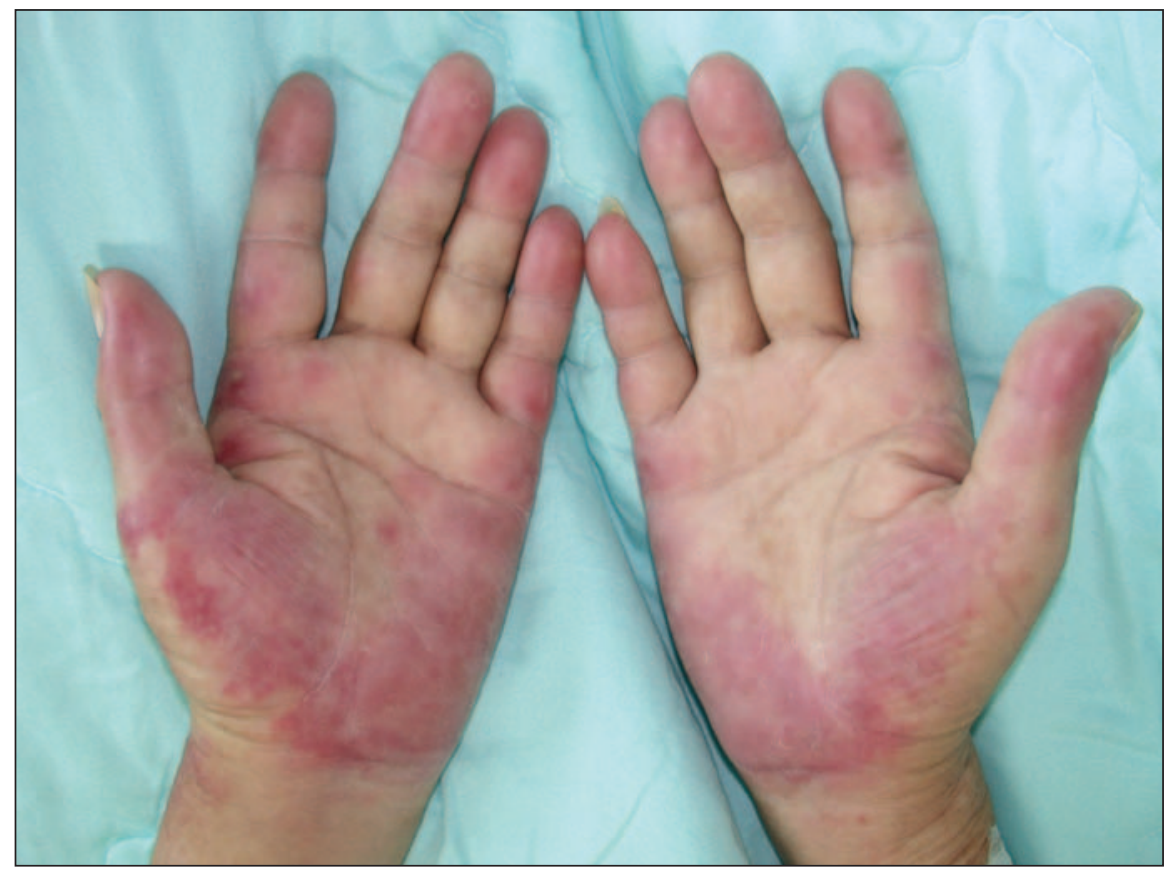

Figure 1: The patient's erythema spread to other areas of his palms and fingers and the skin became edematous 14 days after the start of chemotherapy.

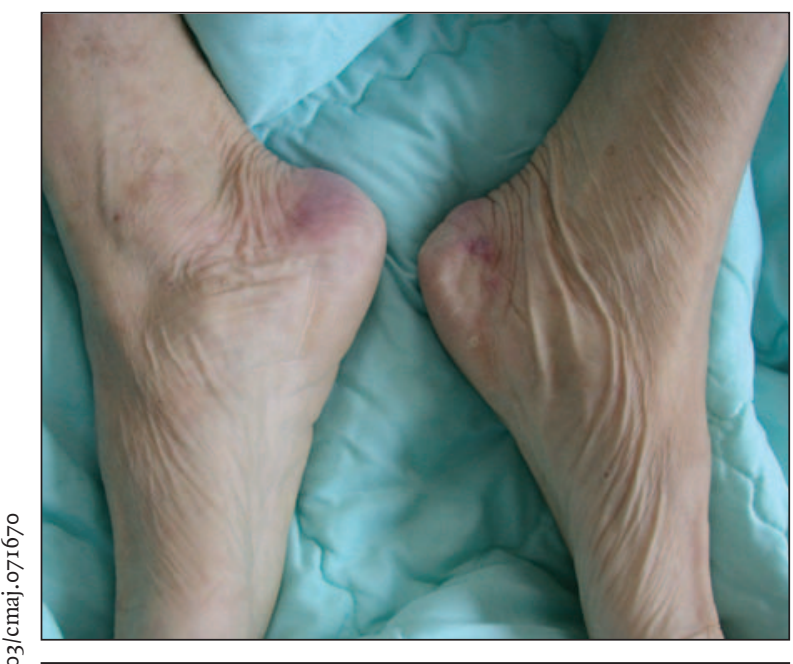

Figure 2: Erythema on the inner sides of both feet near the heels 14 days after the start of chemotherapy.
A 70-year-old man with a spindle-cell malignant tumour located in the left pleura received a first course of chemotherapy that consisted of $20 \mathrm{mg} / \mathrm{m}^{2}$ of epirubicin and $2500 \mathrm{mg} / \mathrm{m}^{2}$ of ifosphamide per day for 3 days, given intravenously. The chemotherapy led to leukopenia with an absolute neutrophil count of 0.06 (normal I.5-8.0) $\times \mathrm{IO}^{9} / \mathrm{L}$. The leukopenia was treated with $300 \mu \mathrm{g}$ of recombinant human granulocyte colonystimulating factor (rhG-CSF) per day for 7 days, given subcutaneously.

Twelve days after the chemotherapy, the patient presented with painful erythema on the thenar and hypothenar eminence of both his hands. On presentation, he was afebrile and had stable vital signs. We found several tender erythematous nodules on his palms. His leukocyte count was 3 I.2 (normal $3.9-10.6) \times \mathrm{IO}^{9} / \mathrm{L}(0.98$ neutrophils, o.or monocytes and o.or lymphocytes). The erythema spread to other areas on his palms and fingers and the skin became edematous 2 days after admission (Figure I). We also found erythema on the inner sides of both feet near the heels (Figure 2).

Based on the patient's history and findings on physical examination, which of the following diagnoses is most likely?

a. Neutrophilic eccrine hidradenitis or palmoplantar eccrine hidradenitis

b. Osler nodes

c. Palmar-plantar erythrodysesthesia syndrome

d. Erythema multiforme

e. Palmar erythema 


\section{WHAT IS YOUR CALL?}

\section{Discussion}

Because the patient had tender, edematous areas isolated to his palms and soles after recent chemotherapy, the answer is (c) palmar-plantar erythrodysesthesia syndrome. This syndrome, also known as hand-foot syndrome and acral erythema, has been described as a dermatologic manifestation of cytotoxic chemotherapy on the palms of the hands and soles of the feet.

Many drugs have been associated with palmar-plantar erythrodysesthesia syndrome, including 5 -fluorouracil, capecitabine, cytarabine, doxorubicin, epirubicin, docetaxel, vinorelbine tartrate and cyclophosphamide. ${ }^{1}$ The condition manifests initially as paresthesia on hands and feet. Painful and edematous erythema then develops within several days of the onset of paresthesia. In severe cases, bullae may form. Desquamation usually follows within weeks of resolution of the erythema. Skin biopsy characteristically shows necrotic keratinocytes and vacuolar changes of the basal layer, as well as papillary dermal edema, dilated blood vessels and perivascular lymphocytic infiltration.

Palmar-plantar erythrodysesthesia syndrome is treated by decreasing the dose or stopping treatment with the offending chemotherapy drugs. Other treatment includes the use of vitamin B6 (pyridoxine) or analgesics, as well as cooling the hands and feet, applying moisturizer and avoiding excessive pressure or friction on the skin. ${ }^{2}$ In our patient, the erythema on the palms and feet disappeared progressively over I week. Desquamation occurred after the patient took $40 \mathrm{mg}$ of vitamin $\mathrm{B} 6$ orally per day for 2 weeks, applied moisturizer and avoided excessive use of his hands and feet.

Differential diagnosis: Table I outlines the possible causes of the patient's condition.

Neutrophilic eccrine hidradenitis is a rare inflammatory skin disorder characterized by erythematous and edematous papules, plaques or nodules located on the trunk, extremities and face. The lesions are usually asymptomatic but may be pruritic or tender. The distinctive histopathologic feature indicating neutrophilic ecrine hidradenitis is a neutrophilic infiltrate around eccrine glands and coils. The condition occurs most often in patients undergoing chemotherapy for acute myelogenous leukemia. It can also occurs in patients with Hodgkin's lymphoma and solid tumours.

Palmoplantar eccrine hidradenitis is another skin disorder with painful erythematous papules and nodules of abrupt onset on the soles and, less frequently, on the palms. Histopathologic findings are similar to those seen in neutrophilic eccrine hidradenitis; however, palmoplantar eccrine hidradenitis appears almost exclusively in otherwise healthy children.

Osler nodes are painful, red, raised lesions that are usually found on the pads of fingers or toes and occasionally on the thenar eminence. They are also a skin manifestation of infective endocarditis. The pathogenesis of Osler nodes is unclear. They may be caused by immune complex deposition or septic microemboli.

Erythema multiforme is an acute, self-limited skin disease. Lesions usually appear symmetrically on the dorsal surfaces of the hands and extensor aspects of the extremities. They may also appear on the palms or soles. The erythematous macules or papules evolve into classic "iris" or "target" lesions, with a central dusky or darker red area and a peripheral paler pink or edematous zone. Patients may experience a burning sensation in affected areas.

Palmar erythema is nontender reddening of the palms of the hands at the thenar and hypothenar eminences. It may also involve the thenar eminence and fingers, and the soles of the feet (plantar erythema). This disorder is associated with various physiologic or pathologic changes, including liver disease, pregnancy, thyrotoxicosis and rheumatoid arthritis. Palmar erythema in liver disease and pregnancy may be due to high levels of estrogen.

\section{Hsi-Hsun Lin MD \\ Jiun-Nong Lin MD}

Division of Infectious Diseases

Department of Internal Medicine

E-Da Hospital/I-Shou University

Kaohsiung County, Taiwan

This article has been peer reviewed.

Competing interests: None declared.

\section{REFERENCES}

I. Webster-Gandy JD, How C, Harrold K. Palmar-plantar erythrodysesthesia (PPE): a literature review with commentary on experience in a cancer centre. Eur J Oncol Nurs 2007;1I:238-46.

2. Hui YF, Giles FJ, Cortes JE. Chemotherapy-induced palmar-plantar erythrodysesthesia syndromerecall following different chemotherapy agents. Invest New Drugs 2002;20:49-53.

$C M A J$ invites contributions to the What is your call? column, which uses multiple-choice questions to guide a focused image-based discussion of the diagnosis or management of clinical cases. Submit manuscripts online at http://mc.manuscriptcentral.com/cmaj. 